\title{
DIMENSIONAL CHANGES CAUSED IN GLASS BY HEATING CYCLES ${ }^{1}$
}

\author{
By A. Q. Tool, D. B. Lloyd, and G. E. Merritt
}

ABSTRACT

In obtaining expansivity curves for a medium flint glass, samples of the glass were tested which had previously received one or another of several different heat treatments, and in these tests heating cycles were employed which made it possible to determine the magnitude of the so-called "permanent" changes in length which are caused either by the annealing or the "disannealing" which may result from the heat treatment imposed on the glass by the heating cycle employed. Annealing decreases the length and specific volume, while disannealing increases them. It is shown that these effects are related to certain icepoint shifts observed in thermometers, and to other effects sometimes observed in various types of glassware.

\section{CONTENTS}

Page

I. Introduction

II. Experimental methods and apparatus. 631

1. General character of the glass tested... 631

2. Heat treatment of samples.... 634

3. Preparation of test specimens

4. The furnace.

5. The temperature measurements

6. The measurement of fringe displacements

7. The heating cycles

III. Experimental results

IV. Concluding discussion

\section{INTRODUCTION}

In common with a great many other materials glass will generally show, as a result of cyclic heat treatments extending over considerable temperature ranges, more or less permanent changes in its specific volume as well as in its form. In causing such effects it is not always necessary that the treatments reach temperatures in the so-called annealing and softening ranges since detectable changes in the specific volume (when determined at any one temperature) are at times produced by treatments which do not reach temperatures much above those of the atmosphere. The changes resulting at such low temperatures in well-annealed glass are, however, small and of little consequence when compared with those which are obtainable only at higher temperatures or which are the result of a failure of the glass to have reached the proper state of physico-chemical equilibrium when it was annealed. These dimensional changes produced by subjecting glasses to heating cycles approaching the annealing range are the subject of this investigation. 
Certain of the well-known fluctuations and displacements common. in the ice points of mercury-in-glass thermometers undoubtedly result from these changes in specific volume. ${ }^{2}$ To introduce the subject of this paper more definitely, a discussion of these ice-point shifts and their possible causes will, therefore, be included here.

Of the ice-point shifts generally observed, part of the smaller ones (especially those occurring without heating above relatively low temperatures) may be traceable to changes in the shape of the bulb which result from variable strains. Strains introduced during the formation of the bulb and which remain effective because the annealing is insufficient may be of this character since they usually change slowly. Semipermanent or variable strains may also develop in an annealed bulb during the actual use of a thermometer in temperature ranges never reaching the annealing range if, for any reason, the expansivity and the possible specific volume change are not uniform over all parts of the glass or glasses used in making the instrument. These nonuniformities may arise from striae, the use of two different glasses, differences in the amount of working the glass receives when fused, or from temperature gradients during the fusions and annealing. Ice-point shifts from such causes should, however, generally be small and it would seem that they might with almost equal probability be either positive or negative. Furthermore, it would seem that while they can not be eliminated entirely a proper choice of glasses and suitable methods of producing both the glass and thermometers should make their significance negligible.

Another type of ice-point shifts which may occur and which result entirely from uniform changes in specific volume may be divided into two classes. As in the case of those ice-point shifts due to strains, the magnitude of those in the first class to be considered should be small and after being produced should usually decrease more and more slowly with time while the thermometer is held at a practically constant temperature near zero. On the basis of their assumed cause these shifts when observed at the minimum temperature of a cycle will, however, always have the nature of a depression and they will also develop, although their magnitude will usually decrease almost linearly with the extent of the range, after heating and cooling cycles which are within and consequently smaller than the usual $0^{\circ}$ to $100^{\circ} \mathrm{C}$. testing range. On extending the range to higher temperatures the observed depression should first increase and then decrease. General experience with glass, and other materials as well, suggests that these depressions result after heating and cooling cycles because the specific volume of even well annealed glass, after allowing for the change due to the normal expansivity, changes in a cycle which lags behind the causative temperature cycle impressed on the glass; that is, after a thermometer is heated very rapidly from $0^{\circ}$ to $100^{\circ} \mathrm{C}$., for example, and after its temperature becomes uniform and the expansion of the glass due to its normal expansivity is completed, the bulb, howcver, will still continue to expand slightly at a decreasing rate. This additional expansion, which continues at a constant temperature and which appears to be of sufficient magnitude and to proceed slowly enough even at $100^{\circ} \mathrm{C}$. for observation in thermometers made from

Young, Nature, 41, p. 488; 1890 . In this paper Young, besides ascribing the rise of the ice point to a volume change causeri by the molecules assuming a position of greater stability, also refers to several interesting notes and articles on this subject by himself and others. 
glasses that are subject to relatively largo depressions in this range, corresponds to a slow increase in the specific volume of the glass. On cooling to zero to complete the cycle this increase in volume will persist for a relatirely long time since, in general, all such readjustments proceed more slowly at lower temperatures. Work by a number of investigator's was required in the development of thermometer glasses which were not unduly subject to the depressions which may be ascribed to readjustments of this kind, and thermometers now made from the best of these glasses seldom show, for cycles between $0^{\circ}$ and $100^{\circ} \mathrm{C}$., depressions of more than one-tenth of $1^{\circ} \mathrm{C}$.

When, however, the temperature cycles are not confined to these relatively restricted ranges such as $0^{\circ}$ to $100^{\circ} \mathrm{C}$., but are increased until temperatures near or within the annealing range are approached, the observed ice-point shifts become, in general, much larger. Such shifts are of the second class which may be ascribed to specific volume changes in the glass, and contrasted to the first class they may, depending on circumstances, consist of either depressions or elevations with the latter being far more common in practical thermometry.

These differences between the two classes arise from the fact that the noticeable readjustments possible in difierent temperature ranges are different. Thus at $100^{\circ} \mathrm{C}$, for example, the only noticeably active readjustments in a glass previously held at $0^{\circ}$ are generally all comparable to disannealing processes, although the state of the glass at $100^{\circ}$ is not one of equilibrium for that temperature, but for some much higher temperature; that is, when a thermometer, for example, is annealed and cooled all major readjustments which might produce equilibrium at $100^{\circ} \mathrm{C}$. cease to operate at any appreciable rate long before this temperature is reached. Certain minor readjustments, however, continue through this point and until the glass is nearer to, or reaches zero. On reheating, the minor processes are reversed and disannealing results throughout a considerable range, $G$. (See footnote 3.) This effect alone is noticeable until some temperature range, $G_{1}$, well above $100^{\circ} \mathrm{C}$. is reached where the major processes again operate at a noticeable rate and the annealing readjustment with its usual decrease in specific volume is resumed. If the heating is continued until a range, $G_{2}$, is reached which is above the temperature corresponding to the equilibrium condition produced in the glass by the previous annealing treatment, the majority of the annealing processes will be reversed, and a disannealing somewhat similar to that at $100^{\circ}$ C. With its usual increase in specific volume will again be the predominant effect. If instead of continuing the heating, the thermometer had been held for some time at any temperature below $G_{1}$ and then cooled rapidly, an ice-point depression would have resulted. Treating and cooling in the same manner from any point in $G_{1}$ would have produced an elevation, the magnitude of which would depend on various factors. From $G_{2}$ such treatments would again produce depressions. ${ }^{3}$ All of these effects may be modified by, but they are not the result of, strains; and it is in fact only those ice-point displacements that are not caused by strains and which may be obtained only by heating to temperatures in and above 
$G_{1}$ that correspond to the experimental results on changing the specific volume which are shown in this paper.

In regard to the magnitude of these particular ice-point displacements it is well known that under some conditions shifts amounting to $30^{\circ} \mathrm{C}$. or more are possible even when the heating cycles are extended to temperatures only moderately high. Since density changes of corresponding magnitudes may be obtained by varying the annealing schedules of most glasses it appears that, as indicater in the previous paragraph, these particular shifts are caused mainly by changes in the specific volumes of thermometer glasses. The fact that these large ice-point shifts are more often elevations than depressions is merely a consequence of the ordinary practices followed in the manufacture and use of thermometers; that is, either the scales, and consequently the probable heating cycles to which the thermometers will be subjected when in use, extend to temperatures which are near or within the annealing ranges of the glasses without actually reaching the effective temperatures ${ }^{4}$ of the annealing treatments previously given to the thermometers; or, these annealing treatments were entirely inadequate since they failed to establish those physico-chemical conditions which are reasonably stable at all temperatures below the annealing range. Inadequate annealing is, of course, indefensible but the practice of somewhat overextending the scales may in some very exceptional cases be justified. This practice should, however, be avoided if possible and it should never be followed without providing an adequate annealing which presumably should be given just before filling and graduating the thermometer and at temperatures as near the top of the proposed scale as possible. Normally it would appear that an adequate annealing treatment for a thermometer is one which will produce a physico-chemical condition corresponding closely to one of equilibrium for a temperature not too far above the lower limit of the practical annealing range. This temperature corresponding to the equilibrium condition is defined in footnote 4 as the effective annealing temperature of an annealing treatment and when a thermometer is heated to points above it an ice-point depression will usually be observed, as previously stated.

The fact that a thermometer can not be expected to give a satisfactory performance if it is heated to temperatures which exceed the lower limit of the annealing range is well known, but it does not appear to be so generally appreciated that this limit is considerably lower than those temperatures which may be employed in giving a glass a reasonably thorough and for most purposes a sufficient annealing in a reasonable time. The best means of determining for a given glass a safe upper limit for the scale is, of course, to extend the heating cycles imposed on a comparatively well annealed test thermometer of the glass gradually to higher and higher temperatures until the point is finally reached where a treatment of several hours at the maximum temperatures of the cycles begins to produce ice-point

\footnotetext{
1 The effective temperature of an annealing treatment may be defined as that annealing temperature at which prictically the same effect produced on the physico-chemical condition by the actual schedule employed might have been obtained through an annealing period at that temperaturre adequate to produce physico-chemical equilibrium and by a cooling rate such that no further annealing occurred during the cooling period. The resultant effective tomperatures of ordinary annealing treatments may be either lower or higher than the actual temperature employed. It is usually lower if the temoerature used is high in the annealing range and the cooling rate is low; while it is generally higher if the temperature employed is low and the annealing period is short.
} 
displacements which just approach the specified tolerances for such shifts. ${ }^{5}$ That this apparently self-evident precaution is often neglected is shown by the fact that many thermometers are graduated as far as $50^{\circ}$ or $100^{\circ} \mathrm{C}$. into that more or less indefinite region termed the annealing range. Such thermometers on being used to register these high temperatures can scarcely be expected to meet successfully any reasonable specifications.

That unwarranted requirements, such as this use of thermometers at too high temperatures, are so often imposed on a glass when they could in most cases be obviated merely by making a correct choice from the glasses arailable can only be explained by assuming that there exists in many quarters an insufficient knowledge concerning certain important characteristics of this material. To aid in presenting a clear conception of these characteristics it was planned some years ago to undertake, in connection with the density determinations in progress, an investigation which would involve a series of expansivity measurements on several samples of a glass, each of which was to have received previously a heat treatment considerably different from that of the others.

At that time a tentative diagram similar to Figure 1 was prepared as an aid in choosing desirable treating temperatures and in predicting and interpreting the results of such tests. To some extent this diagram followed the presentation used by Marchis ${ }^{6}$ in his thesis on permanent modifications of glass but with respect to detail it was based chiefly on the experience gained from a study of the particular glass to be used in the tests, on a general knowledge of the changes which are caused in the properties of glass by heat treatment, and on certain generalizations arrived at after a consideration of the expansivity measurements obtained by Peters, Cragoe, Merritt, and others. ${ }^{7}$

Corresponding diagrams may be constructed for any property affected by the changing physico-chemical conditions in any material having characteristics similar to those of glass.

This diagram (see fig. 1) with some modification is included in the present article mainly because such diagrams have at times been very useful. Although it has certain patent defects, they can not be properly remedied until more data are available. It is, however, sufficiently accurate and elaborate to aid in the discussion of the results presented in this paper.

\section{EXPERIMENTAL METHODS AND APPARATUS}

\section{GENERAL CHARACTER OF THE GLASS TESTED}

The glass chosen for these preliminary experiments was a medium fint, B. S. melt 494, that had been used in previous investigations ${ }^{8}$ on the effect of heat treatment of the density, refractivity, etc. This choice was made because it was desired to compare the dimensional

3 A limit so determined will usually be well below that which is determined by noting the temperature at which the internal pressure from the gas over the mercury column causes the bulb to distend. As a matter of fact in the $G_{1}$ range the two effects involved are of opposite sign. With a well annealed thermometer the $G_{1}$ shifts may all be smaller than a reasonable toleraice. In such a case, however, it is likely that the $\mathrm{G}_{2}$ shifts will become excessive long before the depressions due to pressure become marked.

- L. Marchis Les Modifications Permanentes du Verre et le Déplacement du Zéro des Thermomètres, Paris; 1898.

7 Peters and Cragoe, B. S. Sci. Paper No. 393; 1920. Merritt, B. S. Sci. Paper No. $485 ; 1924$. Peters, B. S. Sei. Paper No. $521 ; 1926$.

8 Tool and Hill, Trans. Soc. Glass Techn., 9, p. 185; 1925. Tool, Tilton, and Hill, J. Opt. Soc. Am. \& Rev. Sci. Inst., 12, p. 490; 1926. 


\section{EXPLANATION OF FIGURE 1}

Curve $P Q$ represents the cooling curve (contraction per unit length) of a glass sample cooled so slowly that the glass follows its sequence of physico-chemical equilibrium conditions. At $P$ a cooling rate of many degrees per second may ba relatively slow while at $Q$ a degree in many years may be very rapid when compared to the respective rates at which a glass that is removed to an appreciable extent from the equilibrium conditions corresponding to these points will approach: stability. Curve $P E$ represents the curve of a sample cooled very rapidly from $700^{\circ} \mathrm{C}$. and from an equilibrium condicion corresponding to that or higher temperatures, for example, small particles quenched from $700^{\circ} \mathrm{C}$. in water. For a time the rate of cooling caused by the quenching is insufficient to prevent some drift along $P Q$, but ultimately, near the temperature determined by the crossing of the curve $R$, this drift ceases and the curve proceeds in the direction $E$. The increasing angle which a tangent to curve $P E$ makes with $P Q$ indicates the decrease in the drift as the temperature falls, and the slope of the straight portion $E^{\prime}$ represents the normal expansivity. Likewise, the remaining two solid circles represent water quenchings from the indicated temperatures and from equilibrium conditions at these temperatures. The first temperature is slightly above the range of curve $R$ and before taking the direction $D$ indicating a normal expansivity there is a slight drift in spite of the rapid cooling caused by quenching; but the lower one being below $R$, the drift is inappreciable and the normal expansivity $C$ is followed.

If now a sample is quenched in air from $600^{\circ} \mathrm{C}$. and from a condition represented by the open circle on the equilibrium sequence at that temperature, the physico-chemical condition of the glass will again drift considerably toward the equilibria of lower temperatures until the cooling curve approaches parallelism to the direction $D$ at the approximate position indicated by the curve $M$. On air quenching from $500^{\circ} \mathrm{C}$. with the glass in the condition indicated by the next lower open circle the drift is relatively slight and the curve soon becomes parallel to $C$ without further drift toward $P Q$. Glass in equilibrium conditions corresponding to the two remaining open circles will, on being air quenched from the indicated temperatures, follow, respectively, the normal expansivity slopes $B$ and $A$ without any appreciable drift toward the equilibria of lower temperatures. Samples of glass caused to follow curves $A$ to $\vec{H}$ will have difierent densities; that following $E$ having the least, and that following $A$ the greatest. These limits are intended to show the maximum variation attainable by practical means.

If the samples of glass $A$ to $E$ cooled as described are heated very rapioly; that is, at rates of the order obtained by water quenching, the expansion curves $A A^{\prime} P$ to $E E^{\prime} P$ will be followed. Samples $A, B$, and $C$ will before crossing the curve $P Q$ show no appreciable drift toward the equilibria of lower temperatures, but will, after crossing and becoming considerably superheated, ultimately drift to the equilibria of higher temperatures. This drift, liowever, will not be appreciable until the region limited by $R^{\prime}$ is entered. The samples $D$ and $E$ will, on the other hand, after reaching the region $R$ drift toward the equilibria at lower temperatures until $P Q$ is crossed, then they will superheat relatively little before drifting toward the equilibria of higher temperatures.

If, however, the samples $A$ to $E$, inclusive, are heated at a much lower rate, the expansion curves will be represented by $A A^{\prime \prime}$ to $E E^{\prime \prime}$. In such a case samples $A$ and $B$ will show no appreciable drift toward the equilibria of lower temperatures before $P Q$ is crossed and, after crossing, the drift toward the equilibria of higher temperatures will be inappreciable only until the curve $\mathrm{S}^{\prime}$ is reached. After crossing curve $S$, samples $C, D$, and $E$ will drift toward equilibrium at lower temperatures until $P Q$ is crossed. After crossing $P Q$ a slight superheating will oceur, and then the drift toward the equilibria of higher temperatures will begin.

To picture the significance of the family of curves to which $R, M$, and $S$ and $R^{\prime}$ and $S^{\prime}$ belong, they may be considered as contour lines on the map of a valley of a mountain stream emerging from a llarrow gorge at $P$ and which gradually widens to a broad and relatively level plain between curves $S$ and $S^{\prime}$. The altitudes so indicated would represent the rapidity of the drift toward $P Q$ shown by glass at different temperatures and in conditions corresponding to difierent equilibria, 
changes caused by the heating cycles imposed on the glass samples during the expansivity tests with those results previously secured from the density changes observed in the same glass and already described in the papers cited.

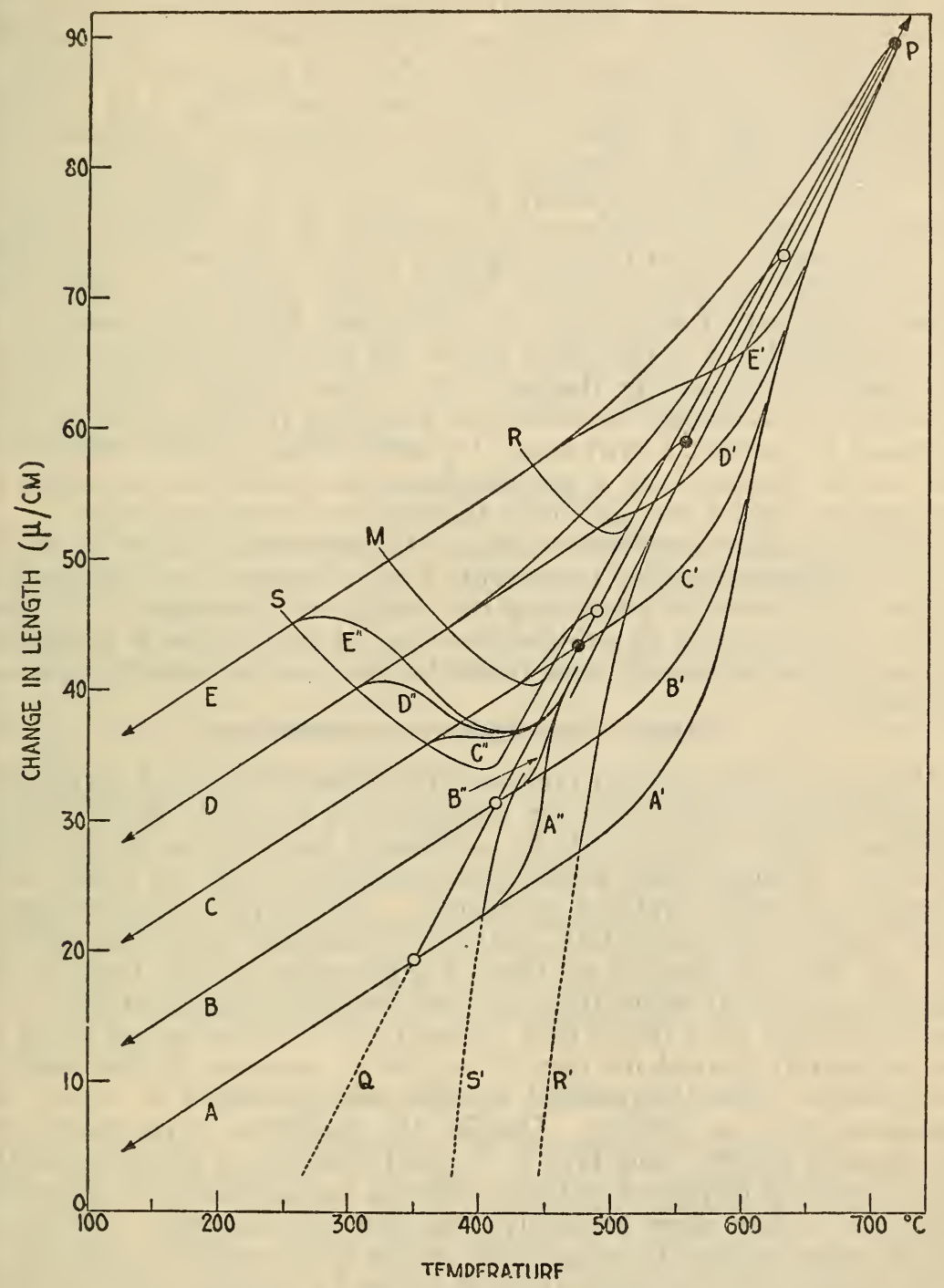

Figure 1.-Diagram showing the effects of heating and cooling conditions on properties of glass

The practical annealing range of this glass lies between the approximate limits $370^{\circ}$ and $470^{\circ} \mathrm{C}$. At the lower temperature the time required for a practically complete relaxation of stresses and the establishment of a physicochemical equilibrium considerably exceeds a month; while at the upper temperature it is of the order of one hour. From the previous investigations it had also been found that the 
original cooling of the pot of glass had been such that the effective annealing temperature was between $400^{\circ}$ and $410^{\circ} \mathrm{C}$. It had also been found that small pieces could be cooled in air at atmospheric temperatures from all temperatures below $420^{\circ} \mathrm{C}$. without introducing permanent stresses materially exceeding the tolerances required for well-annealed glass. Furthermore, below $400^{\circ}$ C. a much slower cooling did not, it appeared, give the glass the opportunity to follow the course of equilibrium conditions (indicated in fig. 1 by the line $P Q$ ) but compelled it, rather, to follow the courses of normal or true expansivity conditions represented by the lines designated by $A, B$, etc. From temperatures above $450^{\circ} \mathrm{C}$., however, chilling in atmospheric air was not drastic enough to prevent all drift along $P Q$ and it was necessary to resort to quenching in water whenever, for example, it was desired to make the glass follow closely the line $C$. Even this drastic treatment fails to halt the drift along $P Q$ if the quenching is from temperatures in the range above the approximate limit $500^{\circ} \mathrm{C}$. This need of increasing the severity of the chilling whenever the quenching temperature is raised and whenever it is at the same time proposed to prevent a drift along $P Q$ reflects the rapidity with which the rate of approaching a physicochemical equilibrium increases as the temperature of the glass rises through the annealing range.

The fixing of the more or less definite temperature limits given above for the different cooling treatments has, of course, no justification except that it aids in presenting the results of experience. In other words, anything but an arbitrary setting of such limits is precluded just as it is in the case of fixing limits for the usual or practical annealing range.

\section{HEAT TREATMENT OF SAMPLES}

Since the intention was to measure the expansivities and permanent dimensional changes on several samples each of which should be in a condition which corresponds to a different position on the line $P Q$, nine lots of small glass pieces were prepared, each lot being large enough to make several test specimens. Eight treating temperatures $\left(360^{\circ}, 370^{\circ}, 390^{\circ}, 400^{\circ}, 420^{\circ}, 440^{\circ}, 470^{\circ}\right.$, and $620^{\circ} \mathrm{C}$.) were chosen, and the heating periods at these temperatures varied from a few minutes at $620^{\circ}$ to more than one and one-half months at $360^{\circ}$. It had previously been found that the required treating period could be approximately halved for each $8^{\circ}$ or $10^{\circ} \mathrm{C}$. increase in the treating temperature. The lot treated at $620^{\circ}$ was quenched in water, the remainder were air chilled. Despite the smallness of the pieces the lots treated at $620^{\circ}$ and $470^{\circ} \mathrm{C}$. drifted during the first part of the cooling toward physicochemical equilibria corresponding to temperatures well below their respective treating temperatures. With the higher temperature the magnitude of the drift probably approached $100^{\circ}$ C., but with the lower $\left(470^{\circ}\right.$ C. $)$ it was much less. For the rest of the samples it is believed the drifts were inappreciable. It was found, however, that the treatment given at $360^{\circ} \mathrm{C}$. was too short to reduce the glass completely to equilibrium at that temperature.

\section{PREPARATION OF TEST SPECIMENS}

As the present investigation was more or less preliminary, variations in the size and shape of the test specimens were tried. The most usual shape, however, was that of a pyramid whose base was so cut 
that it rested on three well-separated feet, thus supplying the stability required of a separator in a sensitive Fizeau interferometer. The surfaces of the feet and the apex were smoothly rounded so that there would not be, at the higher temperatures, an undue deformation at the points of contact between the specimens and the two fused quartz plates used in the interferometer. ${ }^{9}$ In each test a set of three such pyramids all having practically the same height were, of course, needed to serve as separators in supporting the upper plate at the desired distance above the lower one. The final height adjustments required to give suitable interference bands for expansivity measurements by this method have been described in sufficient detail by C. G. Peters and his associates in the articles cited. The height of the pyramids used at different times ranged between 1 and $5 \mathrm{~mm}$, and was in every case carefully measured on a special disk anvil micrometer. In general, the pyramids with broad bases and relatively short altitudes gave the most consistent results; presumably because of two reasons- their greater stability and the closer adherence of their temperature to that of the bottom interferometer plate which, as described later, was used to determine the temperatures.

\section{THE FURNACE}

The interferometer system formed by the quartz plates and these pyramidal test specimens was heated and cooled during the expansivity measurements in a tubular electric furnace similar to that described by Peters and Cragoe. A helix of aluminum tubing buried in the thermal insulation and surrounding the heating coil constituted, however, one modification. Through this tube a flow of some gas or liquid could be used to maintain the desired rate of cooling. Another modification consisted in replacing the customary porcelain cup containing the interferometer by a thick-walled one of aluminum. This cup served better to equalize the temperatures of the interferometer system. The introduction of these aluminum parts restricts the use of the furnace to temperatures below $600^{\circ} \mathrm{C}$., since the melting point of aluminum should not be too closely approached. In other respects these aluminum parts proved satisfactory, and for higher temperatures other metals are available. The heating: and cooling rates employed in the tests recorded here approximated $3^{\circ}$ per minute.

\section{THE TEMPERATURE MEASUREMENTS}

Two methods of determining the temperature were used. In the first, a thermocouple with its hot junction in contact with the base of the aluminum cup containing the interferometer was employed in the usual manner. This served mainly as a control of the heating rates, but it also gave a check on the second method particularly when the temperature was being held constant. The second method consisted of using the lower interferometer plate ${ }^{10}$ as a "refraction thermometer" and was depended upon to give a more accurate determination of the actual temperatures of the test specimens, since they were in direct contact with this plate. In reference to this

${ }^{9}$ Peters and Cragoe, B. S. Sci. Paper No. 393; 1920. Merritt, B. S. Sci. Paper No. 485; 1924. Peters, B. S. Sci. Paper No. 521; 1926. Merritt and Peters, J. Am. Cer. Soc., 9, p. 327; 1926.

io This plate was designed and calibrated for this purpose by Mr. Merritt, who plans in the near future to describe the method more fully. 
plate it will suffice to say that its thickness and the relative inclination of its surfaces were such that it gave a satisfactory set of straight interference fringes which shifted approximately one fringe for each $5^{\circ}$ or $6^{\circ}$ change in temperature. As the movement of these fringes was at all times very regular throughout their length, and since a shift can be estimated to a tenth of a fringe with considerable accuracy, the precision of the temperature determinations was of the order of a half degree. If desired, the top plate may be so designed that a part of it may also be used as a refraction thermometer. In this way the axial temperature gradients existing in the space containing the test specimens may be determined; but the number of fringe systems requiring practically simultaneous attention is then such that a chronograph or other suitable system would be desirable for recording the passage of the fringes.

\section{THE MEASUREMENT OF FRINGE DISPLACEMENTS}

The shifts of the fringe system of the bottom plate and of that of the air wedge determined by the lengths of the test specimens between the two plates were determined by means of the usual equipment which is described in the articles cited. With the exception of a few cases where the test specimens were so tall that the air fringes moved very rapidly past the reference point on the top plate and in such numbers that to have recorded all of them would have been too laborious, the time of practically every such passing of a whole fringe was recorded. As each air fringe count was recorded, the number and fractional part of the refraction thermometer fringes which passed the reference point up to that time was also recorded. When the test specimens were relatively small or the temperature was being held stationary it was occasionally considered advisable to increase the number of observations on the air fringes beyond that of the whole bands. The labor required in recording the increased number of observations is compensated for by the advantage gained when it is found necessary to correct any count which proves to have been erroneous because of the accidental omission of a whole band and also by the greater certainty regarding the trend of the curves in graphical representations of the data. Furthermore, any instability on the part of the interferometer system is also more readily detected by using a larger number of points.

\section{THE HEATING CYCLE}

The upper limit of the heating cycles was chosen with the special purpose of keeping the glass at temperatures where it would not deform appreciably under the weight of the top plate. At the same time it was necessary for the chief purpose in view that this limit should be in the range where a physico-chemical equilibrium would be practically reached in a few hours if the time for procuring the data on a complete cycle was not to be too long. Preliminary tests showed this limit to be both safe and convenient when between $440^{\circ}$ and $445^{\circ} \mathrm{C}$., since at this temperature a large portion of the dimensional changes due to physico-chemical processes were completed within two hours although appreciable deformations were not detected after the heating had been continued for several hours. The heating cycle, therefore, consisted of heating the interferometer 
system, formed by the two plates and three pyramidal spacers all contained in the aluminum cup, at approximately $3^{\circ} \mathrm{C}$., per minute from room temperature to $443^{\circ} \mathrm{C}$., holding it there until the expansion due to physico-chemical changes appeared to be complete, and then of cooling it to the starting temperature at approximately the same rate. At the end of such a run the glass spacers were, in general, either longer or shorter, ${ }^{11}$ depending on the initial condition of the glass. If the effective treating temperature corresponding to this initial condition was below $443^{\circ} \mathrm{C}$, the length of the spacers was increased by the cycle; if it was above, the length was decreased. The magnitude of these changes is controlled chiefly by this difference between the initial effective treating temperature and the holding temperature of the cycle, but it will naturally be reduced if the holding period is insufficient and will be somewhat affected by the changes which progress during the cooling. To insure against insufficient holding periods a second, and sometimes a third, run was made.

\section{EXPERIMENTAL RESULTS}

All details, such as the formula required, the corrections which must be applied because of the air density changes, and so on, which are involved in converting the fringe displacements caused by the changing altitudes of the pyramidal spacers into changes in length per centimeter have been sufficiently described in the articles mentioned above. Figures 2 to 7 represent graphically the changes in length with temperature which were obtained for the differently treated samples. To make sure that the curves shown represented with sufficient fidelity the expansions and contractions which should result from the previous treatments of the samples two or more sets of pyramids from each of the nine lots were tested. Not infrequently there occurred during these runs (usually after the first heating) certain small abrupt fringe displacements which appeared to be in nowise related to the temperature of the glass, and although their combined magnitudes seldom materially affected the general result of any run their occurrence did make repetitions of the tests even more desirable. Such repetitions revealed that these abrupt displacements were entirely accidental and that in most cases they could be either compensated for by taking into account the two or more sets of observations or entirely neglected in deciding upon the most probable trend of the expansion curves. Since the curves in the figures lie so close together, it was found impossible to show the points corresponding to each individual observation and consequently only the smooth trend is given. This procedure does not make these displacements evident, but they were none the less definite.

With regard to the cause of these accidental effects, they seemed to be induced chiefly by the difference in the expansions of the glass pyramids and the fused quartz plates in contact with them. The effect of this difference was probably exaggerated by the slight temperature differences existing between the top and bottom plates. So long as the specimens slipped freely on the plates the differential expansion caused little trouble; consequently, most of the first heat-

11 Schonborn, Sprechsal, 61, p. 99; 1928. This author has published considerable data on the shortening of chilied glass rods. 
ing after the exposure of the interferometer to ordinary atmospheric conditions was accomplished without any marked irregularities in movement. These were likely to appear, however, as soon as the heating had cleared away the possible lubricating films of gases, etc. They were, therefore, presumably caused or accentuated by a gradual tilting of the spacers when these did not slip properly on the plates.

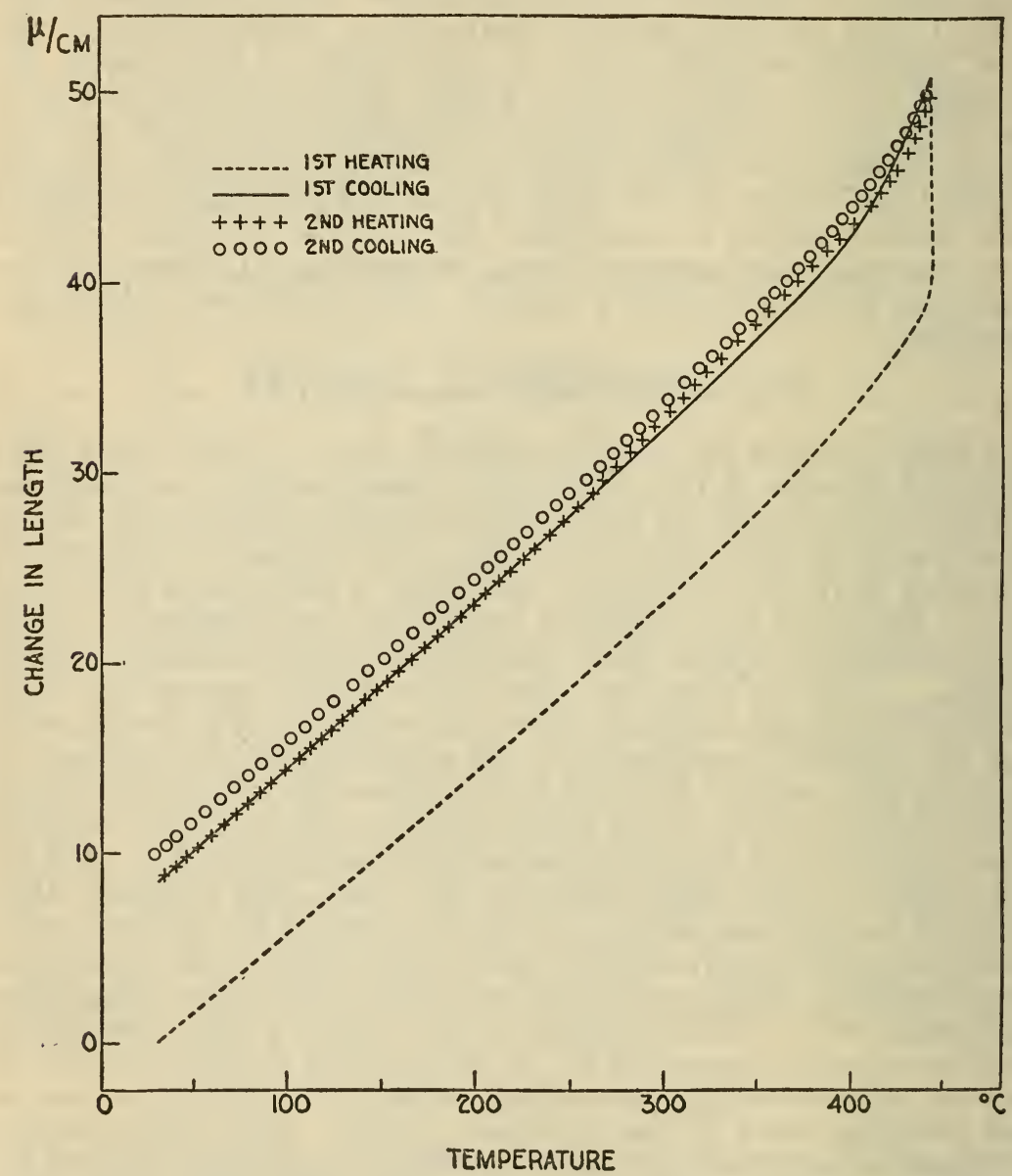

FIgURE 2.-Expansion curves obtained on a sample of glass previously treated at $360^{\circ} \mathrm{C}$. for 47 days and cooled in air

First cycle.-Heating curve indicated by broken line, cooling curve by continuous line. Held at maximum tempcrature approximately one hour.

Second cycle.-Heating curve indicated by crosses, cooling curve by circles. Held at maximum temperature approximately 15 minutes.

The unit $\mu$ (one micron), used in the ordinates, is $10^{-4} \mathrm{~cm}$.

This action changed for a time the apparent rate of expansion which was in most cases ultimately compensated for by a return of the interferometer system to stability. At times this return would be abrupt; at others it was gradual, often causing the apparent expansion curve to show changes in slope which might be erroneously interpreted as indicating some change in the glass. These erratic 
effects are apt to be very troublesome in obtaining cooling curves whenever the heating has been pushed to temperatures where the spacers adhere ${ }^{12}$ slightly to the fused quartz plates. This occurs to some degree with smooth plates, but much more of ten and to a greater extent with plates which have become somewhat roughened and thus allow the glass to fit into and grip their surfaces.

The curves in Figure 2 show essentially how the glass of the sample previously treated at $360^{\circ} \mathrm{C}$. follows, on the first heating, a normal

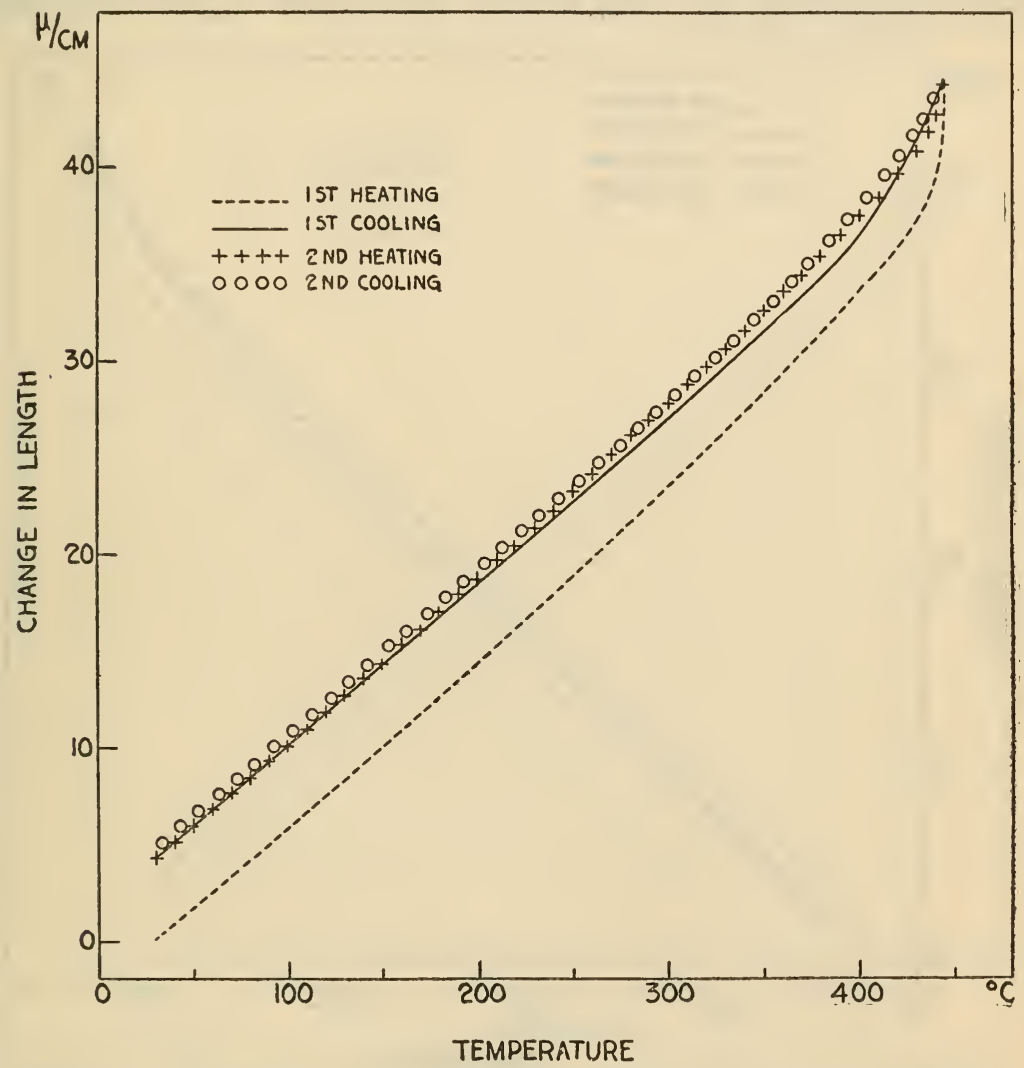

FIGURE 3.-Expansion curves obtained on sample of glass untreated except for the usual cooling in the pot

For description of cycles see remarks under Figure 2 and in text

expansivity curve (similar to $A$ and $B$ in fig. 1) until the line corresponding to equilibrium, conditions ( $P Q$ in fig. 1 ) is crossed. This crossing probably occurred shortly after reaching $370^{\circ} \mathrm{C}$. because it was found that the treatment at $360^{\circ} \mathrm{C}$. had not been long enough to produce more than the effect of a completely effective treatment at $370^{\circ} \mathrm{C}$. This would mean, in view of the rate of heating employed, that shortly after reaching $370^{\circ} \mathrm{C}$. (possibly near $400^{\circ} \mathrm{C}$.) the apparent expansivity should increase somewhat because of a drifi; toward equilibrium. This drift, which in this case is not very marked until $420^{\circ} \mathrm{C}$. is reached, signifies the introduction of a "permanent"

12 Klemm and Berger, Glastechnische Berichte, 5, p. 405; 1927. 
increase in volume. Although the temperature was maintained practically constant at the holding temperature, $443^{\circ} \mathrm{C}$., the increase in length during the holding period was almost a sixth of the total change which took place due to normal thermal expansion between room temperature and this holding temperature.

Considering the low internal mobility of the glass and its resultant slow change toward equilibrium at this and lower temperatures, the rate of cooling, $3^{\circ} \mathrm{C}$. per minute, is rapid and, consequently, the curve representing the first cooling shows only a relatively small

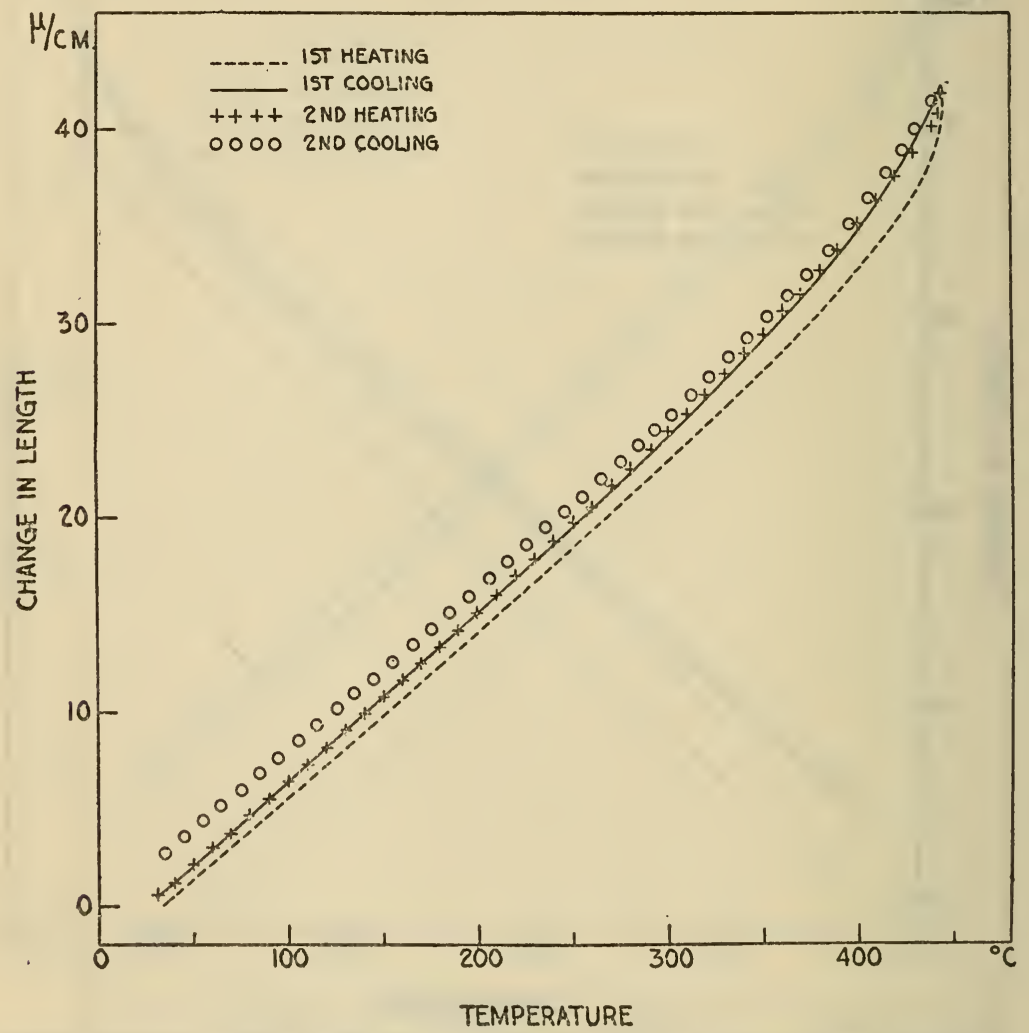

Figune 4.-Expansion curves obtained on sample of glass treated at $420^{\circ} \mathrm{C}$. for five days and cooled in air

For description of cycles seo remarks under Figure 2 and in text

return drift toward any equilibrium for a lower temperature. As a result the major part of the change at the holding temperature was retained until room temperature was reached and this change may, therefore, be termed a permanent one, although it is no more permanent than the opposite change which of necessity preceded it during the annealing treatment of the sample at $360^{\circ} \mathrm{C}$. The second cycle of heating, holding, and cooling produced a slight additional increase, which was mainly due to the fact that the first holding period was too short. In fact, experience would indicate that when starting with the glass in the condition of this sample both these cyclic treat- 
ments combined were insufficient to produce more than possibly nine-tenths of the change which would normally follow the establishment of complete equilibrium in this particular sample at $443^{\circ} \mathrm{C}$. To attempt to gain the whole change would, however, be too tedious in an investigation of this sort. This is true since the process of approaching equilibrium is aperiodic; that is, it proceeds endlessly but at a continually decreasing rate.

Figure 3 represents the results obtained on specimens from the untreated sample. This glass was in a condition corresponding to

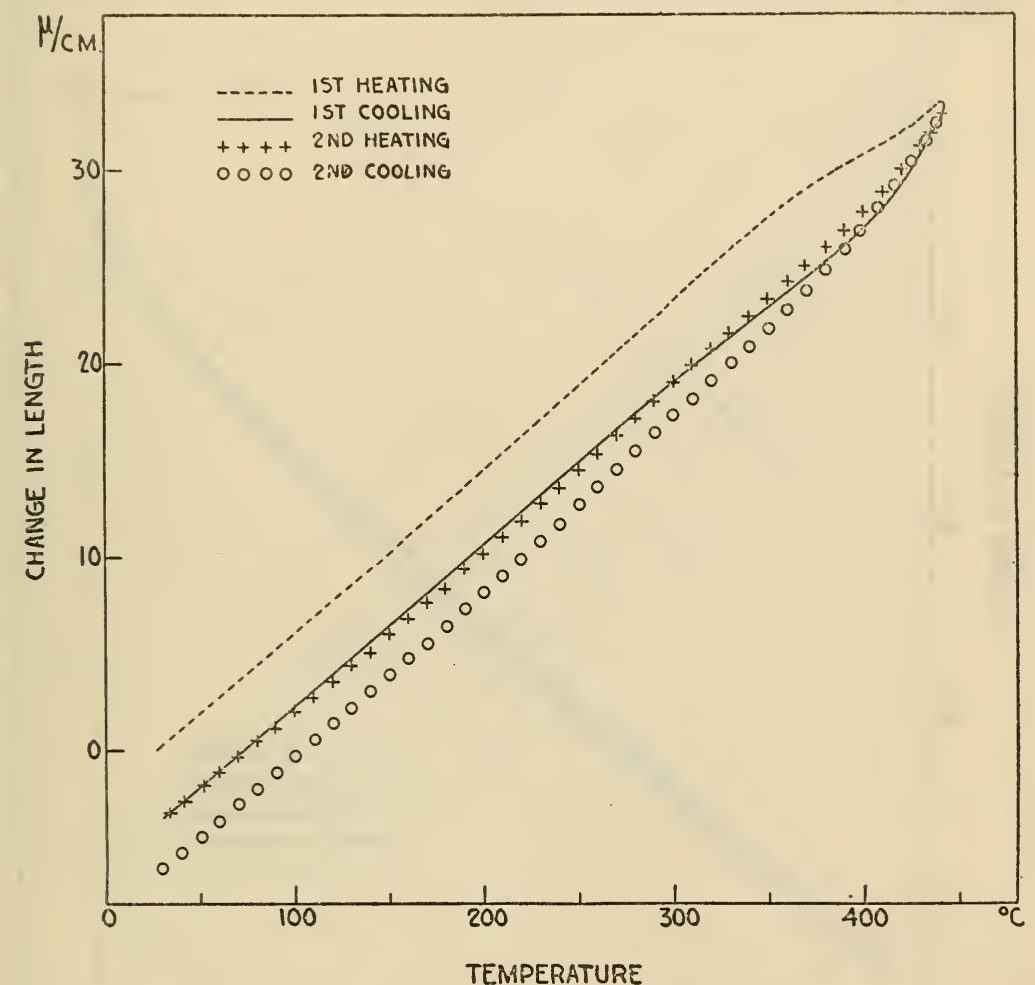

Figure 5.-Expansion curves obtained on sample of glass treated at $470^{\circ} \mathrm{C}$. for three hours and cooled in air

For description of cycles see remarks under Figure 2 and in text

equilibrium at a temperature slightly over $400^{\circ} \mathrm{C}$., consequently, in this case also a noticeable increase in the rate of expansion did not occur until $420^{\circ}$ was reached. The change occurring at the holding temperature was not so great as in the previous case, but, as before, the second cycle increased this permanent change slightly.

The rext case was of interest because it had been previously estimated that the treatment involved in these heating and cooling cycles of the expansivity tests should be approximately the equivalent of an annealing treatment which would produce equilibrium at $420^{\circ} \mathrm{C}$. This appears not to have been entirely correct, since the curves in Figure 4 (see also curve $E$, fig. 7 ) appear to show that $430^{\circ} \mathrm{C}$. would 
have been a closer estimate, a conclusion supported by the curves obtained for the sample treated at $440^{\circ}$ and represented in Figure 7 by curve $C$. In Figure 4 the permanent change in length after the second heating was unduly large in comparison to that found after the first. This may be due to either or both of two causes-either the holding period of the first cycle may have been too short or there may have been a slight difference between the holding temperatures. The latter appears more probable, since to stop a steady rise in tem-

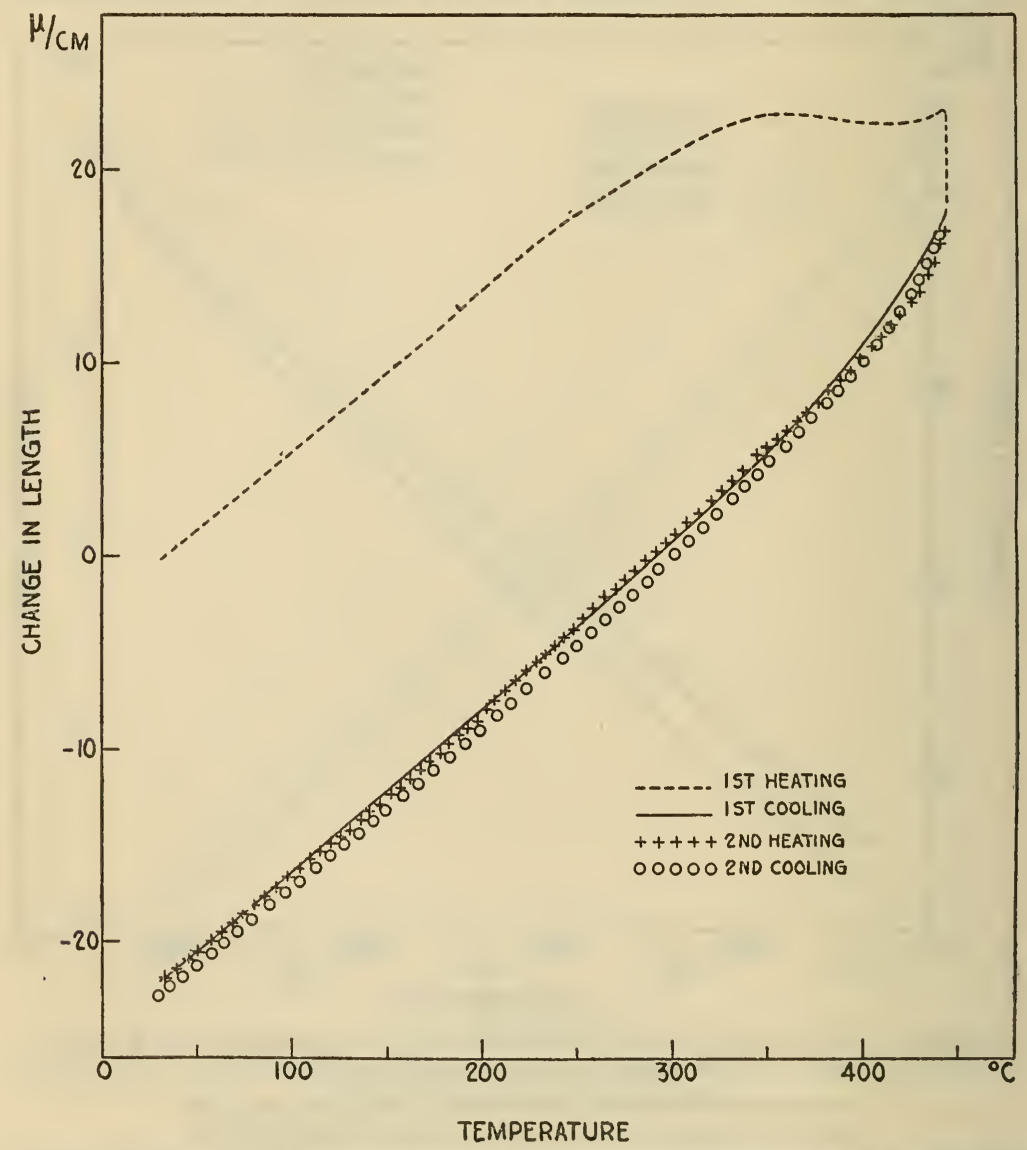

Figure 6.-Expansion curves obtained on sample of glass treated at $620^{\circ} \mathrm{C}$. for 15 minutes and quenched in water

For description of cycles see remarks under Figure 2 and in text

perature of $3^{\circ} \mathrm{C}$. per minute abruptly at a given point without error requires, to say the least, considerable manipulative skill.

In Figure 5, which represents the results obtained on the sample treated at $470^{\circ} \mathrm{C}$., the condition was reached, as was also the case of that treated at $440^{\circ} \mathrm{C}$., where there was no actual crossing of the equilibrium sequence PQ (which if incorporated in this figure should lie to the right) by the expansion curves; that is, the equilibrium temperature established by the treatment of the sample was above the 
holding temperature employed in these expansivity tests. In consequence the specimens from this sample suffered permanent contractions after both cycles. The contraction began at temperatures as low as the $350^{\circ} \mathrm{C}$., on the first heating. In measurements on samples such as these which have been chilled rather severely in the attempt

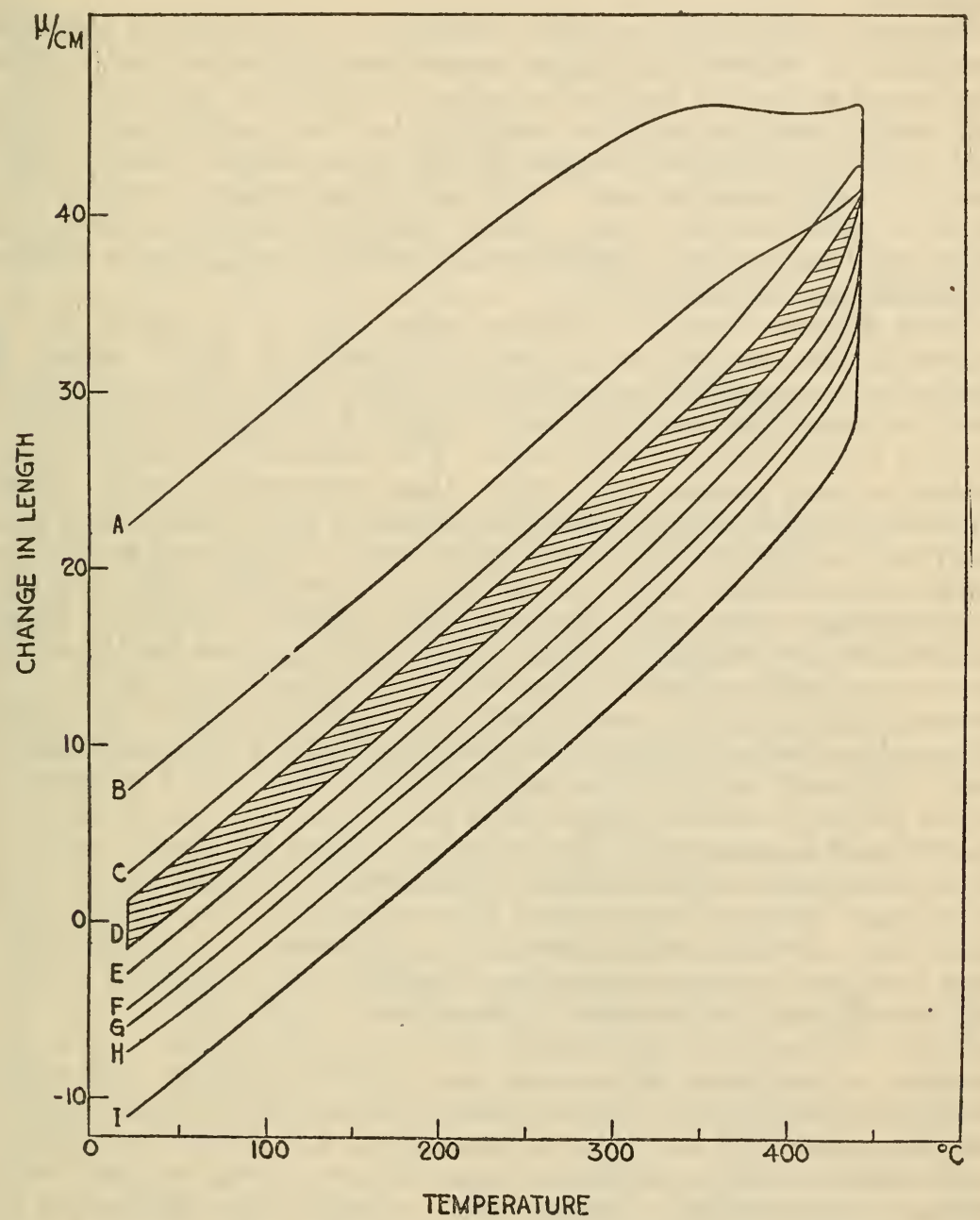

FIGURE 7.-Composite diagram of the expansion curves obtained

Curves $A, B, C, E, F, G, H$, and $I$ represent the first heating curves obtained on the samples previcusly treated at $620^{\circ}, 470^{\circ}, 440^{\circ}, 420^{\circ}, 400^{\circ}, 390^{\circ}$, and $370^{\circ} \mathrm{C}$. The sample corresponding to curve $E$ was untreated. The first cooling curves coincide roughly with the edge of the shaded portion $D$ nearest the corresponding first heating curve. The cooling curves of the second cycle coincide approzimately with a median line through the shaded portion.

to obtain a condition at room temperature which will correspond to a position well up on the sequence PQ (see fig. 1) mechanical strains introduced by this treatment will usually be quite troublesome, since they add considerably to the instability of the interferometer system and produce small disturbing displacements similar to those previously 
discussed. The effects produced by the strains, however, are almost as likely to cause an apparent expansion as they are an apparent contraction. Furthermore, if the test specimens are properly designed, these apparent length changes are, in general, very much smaller than the permanent contractions due to changing equilibrium conditions.

Figure 6 representing results obtained on the sample ${ }^{13}$ quenched from $620^{\circ} \mathrm{C}$., shows a still more pronounced permanent contraction. In this case it appears to have begun below $250^{\circ} \mathrm{C}$. on the first heating, and reached such proportions near $350^{\circ} \mathrm{C}$. that it overshadowed the normal expansivity and changed it into an apparent negative coefficient for rising temperature. At the holding temperature this contraction continued for some time, and practically stopped only when physico-chemical equilibrium at $443^{\circ} \mathrm{C}$. was, from a relative standpoint, not far removed.

From Figures 2 to 6 it will be noted that, as indicated by the curves for the second cycle, the total change in length caused by the normal expansivity on cooling from $443^{\circ} \mathrm{C}$. to room temperature was in all cases practically the same; that is, it was in the neighborhood of 41 or 42 microns per centimeter. This contraction caused by the cooling of the second cycle over this temperature range should obviously be the same for all of the samples, provided the first cycle was practically sufficient to produce in every case a normal equilibrium condition at $443^{\circ} \mathrm{C}$. In view of this, it was possible with very little adjustment to construct a diagram which includes curves for all the various samples and in which all of the cooling curves for the second cycles practically coincide. Figure 7 is such a diagram and in it the curves $A, B, C, E, G, H$, and $I$ correspond, respectively, to the results obtained on the first heatings of specimens from the samples treated at $620^{\circ}, 470^{\circ}, 440^{\circ}, 420^{\circ}, 400^{\circ}, 390^{\circ}$, and $370^{\circ} \mathrm{C}$. Curve $F$ corresponds to the original untreated glass, while the shaded portion, $D$, is so outlined that it is bounded in the majority of cases by the curves of the first coolings and contains those of the second heatings and coolings. It will also include the curves of all succeeding cycles so long as the particular schedule used in these tests is followed. It will be evident that this whole diagram may be considered as occupying an area which in Figure 1 is bounded approximately by the lines $A, A^{\prime \prime}, C^{\prime \prime}$, and $C$. It must be remembered, however, that Figure 1 was constructed on the basis of more or less indefinite heating and cooling conditions and through the use of assumed values for the constants of the glass; that is, it is merely a generalization in which the constants were chosen mainly according to convenience so long as they did not appear unreasonable. Consequently, a perfect correspondence can not be expected, and for that reason the agreement obtained seems striking.

\section{CONCLUDING DISCUSSIOIN}

The permanent changes in length noted after the test specimens from the various samples had been subjected to these treating cycles are indicated in Figure 7 by the ordinate differences at $20^{\circ} \mathrm{C}$. between a central position in the shaded portion, $D$, and the curves $B, I$, etc. Taking into consideration all the determinations obtained on the

3 This was part of the quenched sample used in a previous investigation, the results of which are being prepared for a future publication. 
samples treated between $370^{\circ}$ and $470^{\circ} \mathrm{C}$., it was found that the indicated total permanent change in length which could be produced in this particular glass between these treating temperatures was between 17 and 18 microns per centimeter. While the magnitude of such a change can not be determined by this method with the precision reached when the corresponding volume change is obtained from the determinations required in careful density measurements, it should still approximate the linear change which may be computed from these more precise volume determinations and which had previously been found to be very nearly 16 microns ${ }^{14}$ per centimeter for a difference of $100^{\circ} \mathrm{C}$. in the treating temperatures. That this more precise value is lower than that above is not surprising, since the density changes were determined on large samples which at high treating temperatures allow, because of their size, a relatively larger drift along the equilibrium sequence than that which was permitted by the smaller and more easily quenched samples used for this investigation. One factor leading to greater precison in the density measurements arises from the fact that changes in shape have no effect on such measurements while they always present difficulties in linear expansivity tests.

This medium flint is, of course, not a thermometer glass, although tests have shown that its annealing range approximately coincides with the lead glasses often used in thermometer stems. For that reason it is interesting to compute the ice-point displacements which might be expected if both the bulb and stem were made from it. Since the linear change per degree is approximately $1.6 \times 10^{-5}$ it is seen that in round numbers $5 \times 10^{-5}$ may be considered as the volume change per degree change in the effective annealing temperature. As the differential expansion per ${ }^{\circ} \mathrm{C}$. for this glass and mercury would be about $1.5 \times 10^{-4}$, this means that for every degree reduction in the effective annealing temperature there will be a rise of $1 / 3^{\circ} \mathrm{C}$. in the ice point. If such a thermometer were carefully annealed at $400^{\circ} \mathrm{C}$. before being filled and calibrated to that point, it would later, on being held for a few hours at $390^{\circ} \mathrm{C}$., which is presumably a safe point on the scale, considering that it extends to $400^{\circ} \mathrm{C}$., show a rise which might well exceed any tolerance ordinarily required. Actual tests have shown that some of the $400^{\circ} \mathrm{C}$. thermometers on the market behave in just this manner. In fact, holding them in the range between $380^{\circ}$ and $390^{\circ} \mathrm{C}$. for two weeks will, occasionally at least, produce displacements which exceeds $+10^{\circ} \mathrm{C}$. If the ice point is determined both before and after use at these high temperatures, such a change, since it occurs slowly is, of course, not so important and becomes a mere nuisance. As a matter of fact, however, these thermometers should not be graduated higher than $350^{\circ} \mathrm{C}$.; and, moreover, not to that point unless they have been so carefully annealed that the effective treating temperature has been brought well below $400^{\circ} \mathrm{C}$. Reducing the effective annealing temperature below the upper limit of the scale is of little benefit in the case of such thermometers if they are graduated to $400^{\circ} \mathrm{C}$., because if this temperature has been reduced to $380^{\circ} \mathrm{C}$., for example, any extended use of them at $390^{\circ} \mathrm{C}$. will then produce a considerable ice-point depression.

14 The approximate $\nabla$ alues for the change in density per ${ }^{\circ} \mathrm{C}$. decrease in the effective annealing temperature, the density and the linear expansivity are respectively, $16 \times 10^{-5}, 3.35 \mathrm{~g}^{-\mathrm{cm}^{3}}$, and $0.9 \times 10^{-5} \mathrm{per} \mathrm{C}^{\circ}$. See Tool and Hill, Trans. Soc. Class Tech., 9, p. 199; 1925. 
Using a low annealing range glass of this sort in the stem and a glass with the same expansivity but a higher annealing range in the bulb in order to reach $400^{\circ} \mathrm{C}$. has possibly, in relation to this particular type of ice-point displacement, some advantages but is also likely to cause difficulties of a somewhat different character. These will arise from the fact that it is impossible to anneal both glasses thoroughly, since to properly anneal the bulb would endanger the stem through softening; while if the stem alone is well annealed there will be throughout the annealing period a clamping effect upon the bulb at the line of fusion. This effect caused by the greater shrinkage of the stem will be difficult to eliminate if it is introduced by annealing at low temperatures; and as soon as the thermometer is used at temperatures above the effective annealing point of the stem, the consequent disannealing will reduce the effect and it may even introduce a temporary spreading effect. Such distortions of the bulb would naturally lead to erratic results, especially if the thermometer is employed at too high temperatures.

These particular clamping and spreading effects which appear at junctions and which result, during heating and cooling, from differences in annealing temperatures, have a very wide significance wherever two or more glasses, which may even be only slightly different, are required in fashioning an article. In fact, it is evident that besides the normal expansivities two other factors, the expansions (or contractions) caused by changes in physico-chemical equilibrium conditions and the annealing ranges, must be considered whenever two glasses are fused together. Even in an article made entirely of one glass the first of these two factors may assume considerable importance as a cause of breakage if the heat history of all parts of the article has not been the same and particularly if the whole article is not properly annealed subsequent to its fabrication. This should be evident from the curves presented, since they show that the "permanent" change may easily exceed a sixth of the normal thermal expansion incurred in heating the glass from room temperature to the annealing range.

It will be noted that the normal expansivity of this glass as indicated by the slopes of the curves below the annealing range does not appear to vary greatly and that changes in the heat treatment do not affect it materially. What might seem to be a large variation at the higher temperatures has been shown to be due mainly to the "permanent" dimensional changes which have been the subject of this article and which are not related to the true expansivity of the glass. Any change in the equilibrium condition of the glass should cause, however, some change in the true expansivity, but normally this change in the expansivity is not likely to be more than a few per cent. In fact, $i t$ is to be presumed that the total effect which any such change in this coefficient might cause in cooling from the annealing range to room temperature would normally be less than the "permanent" change in length which is possible as a result of changing the equilibrium temperature through a relatively small interval.

Washington, June 19, 1930. 